\title{
Light Scattering Study of Semiflexible Polymer Solutions III. Multicomponent Solutions
}

\author{
Takahiro Sato, Yuji Jinbo, ${ }^{\dagger}$ and Akio Teramoto* \\ Department of Macromolecular Science, Osaka University, \\ 1-1 Machikaneyama-cho, Toyonaka, Osaka 560-0043, Japan \\ * Research Organization of Science and Engineering, Ritsumeikan University, \\ 1-1-1 Nojihigashi, Kusatsu, Siga 525-8577, Japan
}

(Received September 30, 1998)

\begin{abstract}
The Rayleigh ratio $R_{\theta}$ for multicomponent solutions containing stiff-polymer and small-molecular components of arbitrary concentrations is formulated using the scaled particle theory for wormlike spherocylinders combined with the generalized Ornstein-Zernike integral equation. The general expression for $R_{\theta}$ obtained is applied to the following specific cases: (1) ternary solutions consisting of two homologous polymer species and a solvent, (2) solutions of polydisperse polymer samples, and (3) ternary solutions containing two solutes of different chemical species (e.g., one polymer dissolved in a mixed solvent). The result for the case of (1) is compared with experimental data for dilute through semidilute solutions containing two different molecular-weight samples of poly $(n$-hexyl isocyanate), a semiflexible polymer.

KEY WORDS Light Scattering / Multicomponent Solution / Semiflexible Polymer / Structure Factor / Wormlike Cylinder Model / Poly $(n$-hexyl isocyanate) /
\end{abstract}

Light scattering is a useful technique to characterize polymer molecules and their intermolecular interactions in solution. When applying this technique to multicomponent polymer solutions, one has to take into account some special effects inherent to multicomponent systems. ${ }^{1,2}$ In order to consider these effects, light scattering theories for multicomponent polymer solutions were proposed by many authors. ${ }^{3-8}$ These theories are mainly concerned with dilute polymer solutions, and give us recipes to determine the true molecular weight, second virial coefficient, and radius of gyration of polymers.

In Part II of this series of papers, ${ }^{9}$ we formulated the Rayleigh ratio $R_{\theta}$ or the light-scattering structure factor for isotropic solutions of monodisperse wormlike spherocylinders of arbitrary concentrations, by the scaled particle theory combined with the generalized Ornstein-Zernike integral equation. The formulated $R_{\theta}$ was shown to be favorably compared with experimental data for dilute through semidilute solutions of a semiflexible polymer, $\operatorname{poly}(n$-hexyl isocyanate).

In the present study, we have extended the previous formulation to multicomponent polymer solutions containing macromolecular and small-molecular components to obtain $R_{\theta}$ valid at arbitrary concentrations. While the present theory is not applicable to solutions of flexible polymers due to approximations used in the formulation, it is utilized to analyze light-scattering data for concentrated multicomponent solutions containing stiff-chain polymers, which include important information to characterize intermolecular interactions among solute components. ${ }^{10}$

\section{FORMULATION}

\section{Rayleigh Ratio at the Zero-Scattering Angle}

Consider a solution consisting of $r$ macromolecular and small-molecular species (solutes) and a primary solvent, and suppose that the solute species $s \quad(=1$, $2, \cdots, r)$ is composed of $N_{0, s}$ identical isotropic scattering (monomer) units; for small-molecular species, the whole molecule is regarded as one scattering unit with $N_{0, s}=1$. By fluctuation theories of light scattering, the excess Rayleigh ratio $R_{0}$ (for vertically polarized incident light) of this multicomponent solution over that of the primary solvent at the zero-scattering angle is related to thermodynamic properties of the solution by ${ }^{2,11}$

$$
R_{0}=(2 \pi / \lambda)^{4} \alpha \mu^{-1} \alpha^{T}
$$

where $\lambda$ is the wavelength of light in vacuum, $\alpha$ is the $r$-dimensional row vector whose element $\alpha_{s}(s=1,2, \cdots$, $r$ ) is the excess polarizability of the monomer unit of species $s$, the superscript $\mathrm{T}$ represents the transpose of the row vector $\boldsymbol{\alpha}$, and $\boldsymbol{\mu}$ is the symmetric matrix whose element is given by

$$
\begin{aligned}
\mu_{s t} & \equiv\left(\frac{\partial^{2} \mathscr{F} / k_{\mathrm{B}} T}{\partial c_{0, s}{ }^{\prime} \partial c_{0, t}{ }^{\prime}}\right)_{T, \mu^{\circ}, c_{0, u^{\prime}}} \\
& =\frac{1}{N_{0, s} N_{0, t}}\left[\frac{\partial}{\partial c_{t}^{\prime}}\left(\frac{\mu_{s}}{k_{\mathrm{B}} T}\right)\right]_{T, \mu^{\circ}, c_{0, u^{\prime}}}
\end{aligned}
$$

with the free energy density $\mathscr{F}$ and the chemical potential $\mu_{s}$ of species $s ; k_{\mathrm{B}} T$ is the Boltzmann constant multiplied by the absolute temperature. In eq $2, c_{0, s}{ }^{\prime}$ and $c_{s}{ }^{\prime}$ are the monomer and polymer number concentrations of species $s$, respectively, and $\mu^{\circ}$ is the chemical potential of the primary solvent. (If species $s$ is a small-molecular component, $c_{0, s}{ }^{\prime}=c_{s}{ }^{\prime}$.) Strictly speaking, the above equations are valid for multicomponent solutions which are in a dyalitic equilibrium with the pure primary solvent under the osmotic pressure depending on the composition of the solutions. ${ }^{* 1,11}$ Here we neglect the pressure dependence of $\mu_{s}$ to apply the equations to multicomponent solutions under a constant pressure.

\footnotetext{
${ }^{\dagger}$ Present address: Graduate School of Engineering, Yamagata University, Yonezawa, Yamagata 992-8510, Japan.

${ }^{*} 1$ In eq $2, \mathscr{F}$ is the characteristic function per unit volume $V$ for systems under constant $T, V$, and $\mu^{\circ}$.
} 
In part I of this series, ${ }^{12}$ we calculated the osmotic compressibility or $R_{0}$ for monodisperse stiff polymer solutions by the scaled particle theory for wormlike spherocylinder solutions incorporated with intermolecular dispersion interactions as a thermodynamic perturbation. The thermodynamic quantities $\mu_{s t}$ for multicomponent polymer solutions can be calculated by the same theory, as mentioned below.

Let us consider a solution of volume $V$ containing $r$ wormlike spherocylinder components; the component $s$ has the contour length $L_{\mathrm{c}, s}$ of the cylinder part, the hard-core diameter $d_{s}$, and the persistence length $q_{s}$, and the solution contains $n_{s}$ molecules of the component $s$. Applying the previous result*2 of the scaled particle theory ${ }^{13}$ for $\mu_{s}$, we have

$$
\mu_{s t}=\frac{1}{N_{0, s} N_{0, t}}\left(\frac{\delta_{s t}}{c_{s}^{\prime}}+\frac{2 M_{s} M_{t}}{N_{\mathrm{A}}} \Gamma_{s t}\right)
$$

where $\delta_{s t}$ is Knonecker's delta, $N_{\mathrm{A}}$ is the Avogadro constant, $M_{s}$ is the molecular weight of species $s$, and $\Gamma_{s t}$ is the apparent second virial coefficient between species $s$ and $t$, which is defined by

$$
\begin{aligned}
\frac{2 M_{s} M_{t}}{N_{\mathrm{A}}} \Gamma_{s t} \equiv & \frac{1}{1-\bar{v} c^{\prime}}\left[B_{s t}+C_{s t} \frac{c^{\prime}}{1-\bar{v} c^{\prime}}+D_{s t}\left(\frac{c^{\prime}}{1-\bar{v} c^{\prime}}\right)^{2}\right. \\
& \left.+E_{s t}\left(\frac{c^{\prime}}{1-\bar{v} c^{\prime}}\right)^{3}\right]+\beta_{\mathrm{w}, s t}
\end{aligned}
$$

In this equation, $c^{\prime}$ is the total number concentration of spherocylinders $\left(=\sum_{s=1}^{r} n_{s} / V\right)$, and

$$
\begin{gathered}
\bar{v}=\sum_{s=1}^{r} x_{s} v_{s} \\
B_{s, t}=2 b_{s t} \rho_{s t}+b_{s t}^{\circ}+v_{s}+v_{t} \\
C_{s t}=\sum_{u=1}^{r} x_{u}\left\{\frac { 2 } { 3 } \left[\left(c_{s t u} \rho_{s t}+c_{s t u}^{\circ}\right)+\left(c_{u s t} \rho_{u s}+c_{u s t}^{\circ}\right)\right.\right. \\
\left.+\left(c_{t u s} \rho_{t u}+c_{t u s}^{\circ}\right)\right]+v_{s}\left(2 b_{t u}+b_{t u}^{\circ}\right) \\
\left.+v_{t}\left(2 b_{u s}+b_{u s}^{\circ}\right)\right\}+v_{s} v_{t} \\
D_{s t}=\sum_{u=1}^{r} \sum_{v=1}^{r} x_{u} x_{v}\left\{\frac { 4 } { 3 } \left[v_{s}\left(c_{t u v} \sigma_{t u}+c_{t u v}^{\circ}\right)\right.\right. \\
\left.+v_{t}\left(c_{s u v} \rho_{s u}+c_{s u v}^{\circ}\right)\right] \\
+\frac{2}{3}\left[v_{s}\left(c_{u v t} \rho_{u v}+c_{u v t}^{\circ}\right)+v_{t}\left(c_{u v s} \rho_{u v}+c_{u v s}^{\circ}\right)\right] \\
\left.+v_{s} v_{t}\left(2 b_{u v}+b_{u v}^{\circ}\right)\right\}
\end{gathered}
$$

and

$$
E_{s t}=\sum_{u=1}^{r} \sum_{v=1}^{r} \sum_{w=1}^{r} x_{u} x_{v} x_{w}\left[2 v_{s} v_{t}\left(c_{u v w} \rho_{u v}+c_{u v w}^{\circ}\right)\right]
$$

with the mole fraction $x_{s}\left(=n_{s} / \sum_{s=1}^{r} n_{s}\right)$ of the component $s$, the spherocylinder volume $v_{s}\left(=\frac{\pi}{4} L_{\mathrm{c}, s} d_{s}^{2}+\right.$ $\left.\frac{\pi}{6} d_{s}^{3}\right)$ of the component $s$, and the parameters defined in Table I for the cases of homogeneous mixtures $\left(d_{s}=d\right.$ for all $s$ ) and heterogeneous mixtures. In eq $6-9, \rho_{s t}$ represents the reduction of the intermolecular excluded volume between the components $s$ and $t$ by the orientation defined by eq 11 of ref 13 , and $\rho_{s t}=1$ in the isotropic state.

In eq 4 , the last term $\beta_{\mathrm{w}, s t}$ represents the binary cluster integral with respect to the soft dispersion interaction between species $s$ and $t$, which is formulated in APPENDIX. The explicit form in the isotropic state is given in Table $\mathrm{I}$, where $\delta_{\mathrm{I}, s t}$ (or $\delta_{\mathrm{I}}$ for homogeneous mixtures) represents the strength of the isotropic dispersion interaction between the components $s$ and $t$. It is noted that $\beta_{\mathrm{w}, s t}$ in eq 4 is just the first thermodynamic perturbation term by the soft dispersion interaction and higher perturbation terms are neglected in eq $4 .^{12}$

\section{Angular Dependence of the Rayleigh Ratio}

In order to formulate the angular dependence of the excess Rayleigh ratio $R_{\theta}$, we have to refer the distribution-function theory of light scattering. In terms of the Fourier transforms $\hat{\omega}_{s}\left(k ; i_{1}, j_{1}\right)$ of the intramolecular distribution function for the monomers $i_{1}$ and $j_{1}$ in the same polymer chain 1 of species $s$ and the Fourier transforms $\hat{h}_{s t}\left(k ; i_{1}, i_{2}\right)$ of the intermolecular total correlation function for the monomers $i_{1}$ and $i_{2}$ in the different polymer chains 1 and 2 of species $s$ and $t$, respectively, $R_{\theta}$ can be written by ${ }^{2}$

$$
\begin{aligned}
R_{\theta}= & \left(\frac{2 \pi}{\lambda}\right)^{4} c_{0}{ }^{\prime}\left[\sum_{s=1}^{r} \alpha_{s}^{2} x_{0, s} \hat{\omega}_{s}(k)\right. \\
& \left.+c_{0}^{\prime} \sum_{s, t=1}^{r} \alpha_{s} \alpha_{t} x_{0, s} x_{0, t} \hat{h}_{s t}(k)\right]
\end{aligned}
$$

Here $c_{0}{ }^{\prime}$ is the number concentration of the total scattering units of all solute species, $x_{0, s}$ is the mole fraction of the scattering units of species $s$ in the total units, and $\hat{\omega}_{s}(k)$ and $\hat{h}_{s t}(k)$ are defined by

$$
\hat{\omega}_{s}(k) \equiv \frac{1}{N_{0, s}} \sum_{i_{1}, j_{1}=1}^{N_{0, s}} \hat{\omega}_{s}\left(k ; i_{1}, j_{1}\right)
$$

and

$$
\hat{h}_{s t}(k) \equiv \frac{1}{N_{0, s} N_{0, t}} \sum_{i_{1}=1}^{N_{0, s}} \sum_{i_{2}=1}^{N_{0, t} t} \hat{h}_{s t}\left(k, i_{1}, i_{2}\right)
$$

( $k$ : the absolute value of the scattering vector). Equation 10 is conveniently written in the following matrix notation:

$$
R_{\theta}=(2 \pi / \lambda)^{4} c_{0}^{\prime}\left[\alpha \hat{\boldsymbol{S}}(k) \alpha^{\mathrm{T}}\right]
$$

where $\hat{\boldsymbol{S}}(k)$ is the $r \times r$ structure factor matrix defined by

$$
\hat{\boldsymbol{S}}(k)=\hat{\boldsymbol{\Omega}}(k)+c_{0}{ }^{\prime} \hat{\boldsymbol{H}}(k)
$$

with two matrices, $\hat{\boldsymbol{\Omega}}(k)$ and $\hat{\boldsymbol{H}}(k)$, whose $s t$ elements are respectively given by

$$
\hat{\Omega}_{s t}(k) \equiv x_{0, s} \hat{\omega}_{s}(k) \delta_{s t}
$$

and

$$
\hat{H}_{s t}(k) \equiv x_{0, s} x_{0, t} \hat{h}_{s, t}(k)
$$

The $s t$ element $\hat{S}_{s t}(k)$ of $\hat{\boldsymbol{S}}(k)$ is referred to as the partial structure factor for species $s$ and $t$.

\footnotetext{
${ }^{* 2}$ Equations 7 and 8 of ref 13 contain errors; $B$ in eq 7 and $B_{s}^{\prime} / 2$ and $C_{s}^{\prime} / 3$ in eq 8 should be replaced by $B / 2, B_{s}$, and $C_{s}$, respectively.
} 
Several workers ${ }^{14-18}$ calculated $\hat{\omega}_{s}(k)$ using the wormlike chain model. Among them, the calculation of Yoshizaki and Yamakawa ${ }^{18}$ is the most accurate in the $k$ range of light scattering. In the following, we use their result for $\hat{\omega}_{s}(k)$.

The function $\hat{h}_{s t}(k)$ may be calculated using the generalized Ornstein-Zernike (GOZ) integral equation. ${ }^{19-24}$ In Part II, ${ }^{9}$ we utilized this equation to formulate $R_{\theta}$ for monodisperse polymer solutions. The extension of the equation to multicomponent polymer solutions may be given by ${ }^{23,24}$

$$
\hat{\boldsymbol{H}}(k)=\hat{\boldsymbol{\Omega}}(k) \hat{\boldsymbol{C}}(k) \hat{\boldsymbol{\Omega}}(k)+c_{0}{ }^{\prime} \hat{\boldsymbol{\Omega}}(k) \hat{\boldsymbol{C}}(k) \hat{\boldsymbol{H}}(k)
$$

or

$$
\hat{\boldsymbol{H}}(k)=\left[\boldsymbol{I}-c_{0}{ }^{\prime} \hat{\boldsymbol{\Omega}}(k) \hat{\boldsymbol{C}}(k)\right]^{-1} \hat{\boldsymbol{\Omega}}(k) \hat{\boldsymbol{C}}(k) \hat{\boldsymbol{\Omega}}(k)
$$

where $\hat{\boldsymbol{C}}(k)$ is the $r \times r$ matrix whose $s t$ element is the Fourier transform $\hat{C}_{s t}(k)$ of the (average) direct correlation function between scattering units of species $s$ and $t$, and $\boldsymbol{I}$ is the unit matrix.

As mentioned in Part $\mathrm{II}^{9}$ the GOZ integral equation includes the following approximations:

(1) It does not consider the intramolecular excludedvolume effect for polymer species, i.e., the interaction between monomer units or interaction sites belonging to a same polymer chain (the self interaction).

(2) It takes into account all single-contact terms in $\hat{h}_{s t}(k)$ for any sizes of interacting clusters, but neglects all multiple-contact terms for interacting clusters where at least a pair of polymer chains interact on more than one pair of interaction sites.

(3) In eq 17 and 18, the Fourier transforms of the polymer-polymer and polymer-small-molecule direct correlation functions are replaced by ones $\hat{C}_{s t}(k)$ averaged along the chain contours. That is, the equations neglect the dependence of those direct correlation functions on the position of the interacting monomer-units along the polymer chains.

Although the self-interaction and the multiple contact in polymer chains play important roles in light scattering behavior of flexible polymer-good solvent systems, ${ }^{2}$ their importance may be much reduced with increasing the polymer chain stiffness. ${ }^{9}$ It is well known that polymer chains with sufficient stiffness take unperturbed conformations even in good solvent. ${ }^{25}$ Therefore, the above approximations (1) and (2) may be good for such stiffchain polymer solutions. Furthermore, if the polymer chain is homogeneous and long enough, the averaging process of the Fourier transforms of the direct correlation functions along the polymer chain contour may not substantially affect the final result of the integral equation.

In addition to the above, we have used the following approximation in eq 17 and 18:

(4) Each small molecular component is regarded as a semiflexible spherocylinder in the scaled particle theory to formulate $R_{0}$ above, so that the direct correlation function concerned with the small molecular component has to be regarded as an orientation-dependent quantity. ${ }^{26}$ To obtain eq 17 and 18, however, those direct correlation functions have been preaveraged isotropically with respect to the orientation of small molecular components, and the orientational dependence has not been explicitly considered.

This approximation is expected not to affect the (isotropic) Rayleigh ratio within the light scattering $k$ region.

Combining eq 14 and 18, we have

$$
\hat{\boldsymbol{S}}(k)^{-1}=\boldsymbol{\Omega}(k)^{-1}-c_{0}{ }^{\prime} \hat{\boldsymbol{C}}(k)
$$

Thus we can calculate the partial structure factor $\hat{S}_{s t}(k)$, if we know $\hat{C}_{s t}(k)$. The direct correlation function has a nature of taking non-zero values only when two scattering units (or interaction sites) concerned approach within their interaction ranges. If we choose monomer units of polymer species and whole small molecules as scattering units or interaction sites, their interaction ranges are too short to detect by light scattering experiments; i.e., the interaction ranges multiplied by the light scattering $k$ are all much smaller than unity. In such a condition, $\hat{C}_{s t}(k)$ can be approximated by $\hat{C}_{s t}(0)$.

Comparing eq 1 with eq 13 , we have the relation

$$
\boldsymbol{\mu}=\left[c_{0} \hat{\boldsymbol{S}}^{(0)}\right]^{-1}=c_{0}{ }^{-1} \boldsymbol{\Omega}(0)^{-1}-\hat{\boldsymbol{C}}(0)
$$

where the second equation is obtained using eq 19. Inserting eq 3 and 15 into eq 20 , we can relate the quantity $\hat{C}_{s t}(0)$ to the apparent second virial coefficient $\Gamma_{s t}$ by

$$
\hat{C}_{s t}(0)=-\frac{2 M_{0, s} M_{0, t}}{N_{\mathrm{A}}} \Gamma_{s t}
$$

where $M_{0, s}$ is the molecular weight of the monomer unit of species $s$. (It is noted that $\hat{\omega}_{s}(k)$ is equal to $N_{0, s}$ at $k=0$.) Therefore, eq 13, 19, and 21 give us the final expression of $R_{\theta}$.

At the end of this section, we should refer to other theories dealing with the same problem. Schweizer and Curro $^{23,24}$ calculated the structure factor for polymer mixtures from the GOZ equation using the closure procedure based on "the mean spherical approximation." Their method may be more rigorous than ours mentioned above, but it needs an involved numerical analysis to solve the integral equation, and the structure factor cannot be expressed in an analytical form. Benoit and Benmouna ${ }^{27}$ also calculated $R_{\theta}$ for solutions of polymer mixtures with arbitrary concentrations by the GOZ integral equation. In their formulation, interactions among monomer units were represented in terms of excluded-volume parameters $\left(\vartheta_{\mathrm{A}}, \vartheta_{\mathrm{B}}, \vartheta_{\mathrm{AB}}\right)$, which are not explicitly specified from the molecular level but treated just as phenomenological parameters. (In fact, their excluded-volume parameters are concentration dependent, though the original authors did not argue their dependence.)

\section{APPLICATIONS TO SOME SPECIFIC SYSTEMS}

Ternary Solutions with Two Homologous Polymer Species

Let us consider first a solution of two stiff-polymer samples (components 1 and 2) of the same kind but with different molecular weights, dissolved in a solvent. These polymer samples have the identical hard-core diameter $d$, the persistence length $q$, the dispersion interaction parameter $\delta$, the excess polarizability $\alpha$, and the monomer molecular weight $M_{0}$. From eq 13,19 , and 21 , we have 


$$
\frac{K c}{R_{\theta}}=\frac{\left[1+2 c w_{1} M_{1} P_{1}(k) \Gamma_{11}\right]\left[1+2 c w_{2} M_{2} P_{2}(k) \Gamma_{22}\right]-4 c^{2} w_{1} w_{2} M_{1} M_{2} P_{1}(k) P_{2}(k) \Gamma_{12}^{2}}{w_{1} M_{1} P_{1}(k)+w_{2} M_{2} P_{2}(k)+2 c w_{1} w_{2} M_{1} M_{2} P_{1}(k) P_{2}(k)\left[\Gamma_{11}+\Gamma_{22}-2 \Gamma_{12}\right]}
$$

where $c$ is the total polymer mass concentration, $w_{s}$, $M_{s}$, and $P_{s}(k)$ are the weight fraction (in the total polymer), the molecular weight, and the intramolecular interference factor $\left(\equiv \hat{\omega}_{s}(k) / N_{0, s}\right)$ of polymer species $s$, respectively, $K$ is the optical constant defined by

$$
K \equiv\left(4 \pi^{2} n^{2} / N_{\mathrm{A}} \lambda^{4}\right)(\partial n / \partial c)^{2}
$$

with the refractive index $n$ of the solution, and $\Gamma_{s t}$ is the apparent second virial coefficient for species $s$ and $t$, given by eq 4 .

\section{Solutions of Polydisperse Polymer Samples}

If $L_{s} / d$ is much larger than unity for all $s, \Gamma_{s t}$ defined by eq 4 are independent of $s$ and $t$, being written as

$$
\begin{aligned}
\Gamma_{2, s t}=\Gamma_{2} \equiv & \frac{\pi d N_{\mathrm{A}}}{4 M_{\mathrm{L}}^{2}}\left\{\frac { 1 } { 1 - \overline { v } c ^ { \prime } } \left[1+4 \frac{\bar{v} c^{\prime}}{1-\bar{v} c^{\prime}}\right.\right. \\
& \left.\left.+5\left(\frac{\bar{v} c^{\prime}}{1-\bar{v} c^{\prime}}\right)^{2}+2\left(\frac{\bar{v} c^{\prime}}{1-\bar{v} c^{\prime}}\right)^{3}\right]+\frac{\delta}{d}\right\}
\end{aligned}
$$

where $M_{\mathrm{L}}$ is the molar mass per unit contour length of polymer. In such a case, the inverse of the structure factor matrix $\hat{\boldsymbol{S}}(k)^{-1}$ can be simplified, and eq 13, 19, and 21 finally yield

$$
K c / R_{\theta}=\left(M_{w} P(k)_{z}\right)^{-1}+2 \Gamma_{2} c
$$

where $M_{w}$ is the weight average molecular weight and $P(k)_{z}$ is the $z$-average intramolecular interference factor defined by

$$
P(k)_{z}=\frac{1}{M_{w}} \sum_{s=1}^{r} w_{s} M_{s} P_{s}(k)
$$

At $\theta=0$, where $P(k)_{z}=1$, the above equation gives $K c / R_{0}$ identical with that for a monodisperse polymer solution with the molecular weight equal to $M_{w}$ and volume fraction equal to $\bar{v} c^{\prime}$. Thus polydispersity in molecular weight does not change the form of the equation for $K c / R_{0}$, if the solution contains only sufficiently high molecular weight stiff-polymer components. The same conclusion was previously obtained for the equation for the concentration gradient in polydisperse stiff-polymer solutions in sedimentation equilibrium experiments. ${ }^{10}$ On the other hand, the angular dependence of $K c / R_{\theta}$ is strongly affected by polydispersity through $P(k)_{z}$.

When the total polymer concentration $c^{\prime}$ of a polydisperse polymer solution is sufficiently low, we can use the second virial approximation. Expanding each element $\left[\hat{\boldsymbol{S}}(k)^{-1}\right]_{s t}$ with respect to $c^{\prime}$ and omitting the higher virial terms, we obtain ${ }^{11}$

$\frac{K c}{R_{\theta}}=\frac{1}{M_{w} P(k)_{z}}+2 c \frac{\sum_{s, t=1}^{r} w_{s} w_{t} M_{s} M_{t} P_{s}(k) P_{t}(k) A_{2, s t}}{\left[M_{w} P(k)_{z}\right]^{2}}$

where $A_{2, s t}$ is the second virial coefficient for the components $s$ and $t$ given by

$$
A_{2, s t}=\left(\Gamma_{s t}\right)_{c=0}=\frac{N_{\mathrm{A}}}{2 M_{s} M_{t}}\left(B_{s t}+\beta_{\mathrm{w}, s t}\right)
$$

with $B_{s t}$ and $\beta_{\mathrm{w}, s t}$ given by eq 6 and in Table I, respectively.

\section{Ternary Solution with Two Solutes of Different Chemical Species}

Next consider a solution contains two different kind solute components 1 and 2 , each of which is a stiffpolymer or a small molecule. In general, the two solute components have different light-scattering powers, which can be expressed in terms of the refractive index increments $\gamma_{s} \equiv \partial n / \partial c_{s}$. Equations 13, 19, and 21 gives the Rayleigh ratio for this system as

$$
\frac{R_{\theta}}{K^{\prime}}=\frac{c_{1} M_{1} P_{1}(k) \gamma_{1}^{2}+c_{2} M_{2} P_{2}(k) \gamma_{2}^{2}+2 c_{1} c_{2} M_{1} M_{2} P_{1}(k) P_{2}(k)\left(\gamma_{2}^{2} \Gamma_{11}-2 \gamma_{1} \gamma_{2} \Gamma_{12}+\gamma_{1}^{2} \Gamma_{22}\right)}{\left[1+2 c_{1} M_{1} P_{1}(k) \Gamma_{11}\right]\left[1+2 c_{2} M_{2} P_{2}(k) \Gamma_{22}\right]-4 c_{1} c_{2} M_{1} M_{2} P_{1}(k) P_{2}(k) \Gamma_{12}{ }^{2}}
$$

where $K^{\prime} \equiv 4 \pi^{2} n^{2} / N_{\mathrm{A}} \lambda^{4}$ and $\Gamma_{s t}$ is given by eq 4 .

\section{EXPERIMENTAL TEST OF EQUATION 22}

In order to test the validity of the above derived equation 22 of $R_{\theta}$ for ternary solutions containing two different molecular weight samples of a homologous polymer, we made light scattering measurements for isotropic dichloromethane (DCM) solutions with two fractionated poly ( $n$-hexyl isocyanate) (PHIC) samples. We have chosen two PHIC samples, K-3 $\left[M_{w}=1.11 \times\right.$ $10^{5} ;\left\langle S^{2}\right\rangle_{2}^{1 / 2}$ (the root-mean-square radius of gyration) $=$ $26.6 \mathrm{~nm}]$ and V-1-2 $\left[M_{w}=1.06 \times 10^{6} ;\left\langle S^{2}\right\rangle_{2}^{1 / 2}=97.9\right.$ $\mathrm{nm}]$, previously used for light scattering study of binary solutions. ${ }^{12}$ DCM solutions of each PHIC sample, separately prepared, were filtrated by Millipore filters directly into a light scattering cell to prepare a ternary solution with a given composition. Light scattering experiment was made at $20^{\circ} \mathrm{C}$ in the same procedure as in the previous study. ${ }^{12}$

Figure 1 shows the plots of $K c / R_{\theta}$ vs. $k^{2}$ for DCM solutions containing the two PHIC samples with three different mixing ratios, which are expressed by $\zeta_{2} \equiv$ $w_{2} M_{w, 2} /\left(w_{1} M_{w, 1}+w_{2} M_{w, 2}\right)$ where the subscripts 1 and 2 indicate the lower and higher molecular weight samples, K-3 and V-1-2, respectively. The angular dependence of $K c / R_{\theta}$ becomes stronger with increasing $\zeta_{2}$, but is almost independent of the total polymer concentration $c$.

Equation 22 contains the following molecular parameters: $L_{1}, L_{2}, q, d$, and $\delta_{\mathrm{I}}$. Among them, $L_{1}$ and $L_{2}$ can be calculated from $M_{w, 1}$ and $M_{w, 2}$ with the molar 
Table I. Parameters appearing in eq 4 and $6-9$

\begin{tabular}{lcc}
\hline Parameter & Homogeneous mixture & Heterogeneous mixture \\
\hline$b_{s t}$ & $\frac{\pi}{4} L_{\mathrm{c}, s} L_{\mathrm{c}, t} d$ & $\frac{\pi}{8} L_{\mathrm{c}, s} L_{\mathrm{c}, t}\left(d_{s}+d_{t}\right)$ \\
$b_{\mathrm{st}}^{\circ}$ & $3\left(v_{s}+v_{t}\right)$ & $\frac{\pi}{4}\left[d_{s}^{2}\left(L_{\mathrm{c}, t}+2 d_{t}\right)+d_{t}^{2}\left(L_{\mathrm{c}, s}+2 d_{s}\right)+2 d_{s} d_{t}\left(L_{\mathrm{c}, s}+L_{\mathrm{c}, t}\right)\right]$ \\
$c_{\mathrm{s} t u}$ & $2\left(v_{u}+\frac{\pi}{12} d^{3}\right) b_{\mathrm{st}}$ & $\frac{\pi^{2}}{8} d_{s} d_{t} d_{u}\left(L_{\mathrm{c}, u}+d_{u}\right) L_{\mathrm{c}, s} L_{\mathrm{c}, t}$ \\
$c_{s t u}^{\circ}$ & $2\left(v_{u}+\frac{\pi}{12} d^{3}\right)\left(v_{\mathrm{s}}+v_{t}-\frac{\pi}{12} d^{3}\right)$ & $\frac{\pi^{2}}{8} d_{s} d_{t} d_{u}\left(L_{\mathrm{c}, \mathrm{u}}+d_{u}\right)\left(L_{\mathrm{c}, s} d_{t}+L_{\mathrm{c}, t} d_{s}+d_{s} d_{t}\right)$ \\
$\beta_{\mathrm{w}, s t}$ & $2\left[b_{s t}+4\left(v_{s}+v_{t}\right)+\frac{2 \pi}{3} d^{3}\right] \frac{\delta_{1}}{d}$ & $\frac{\pi}{2}\left[L_{\mathrm{c}, s} L_{\mathrm{c}, t}+2\left(L_{\mathrm{c}, s}+L_{\mathrm{c}, t}\right)\left(d_{s}+d_{t}\right)+2\left(d_{s}+d_{t}\right)^{2}\right] \delta_{\mathrm{l}, s t}$.
\end{tabular}

$L_{\mathrm{c}, s} \equiv L_{s}-d_{s}$.
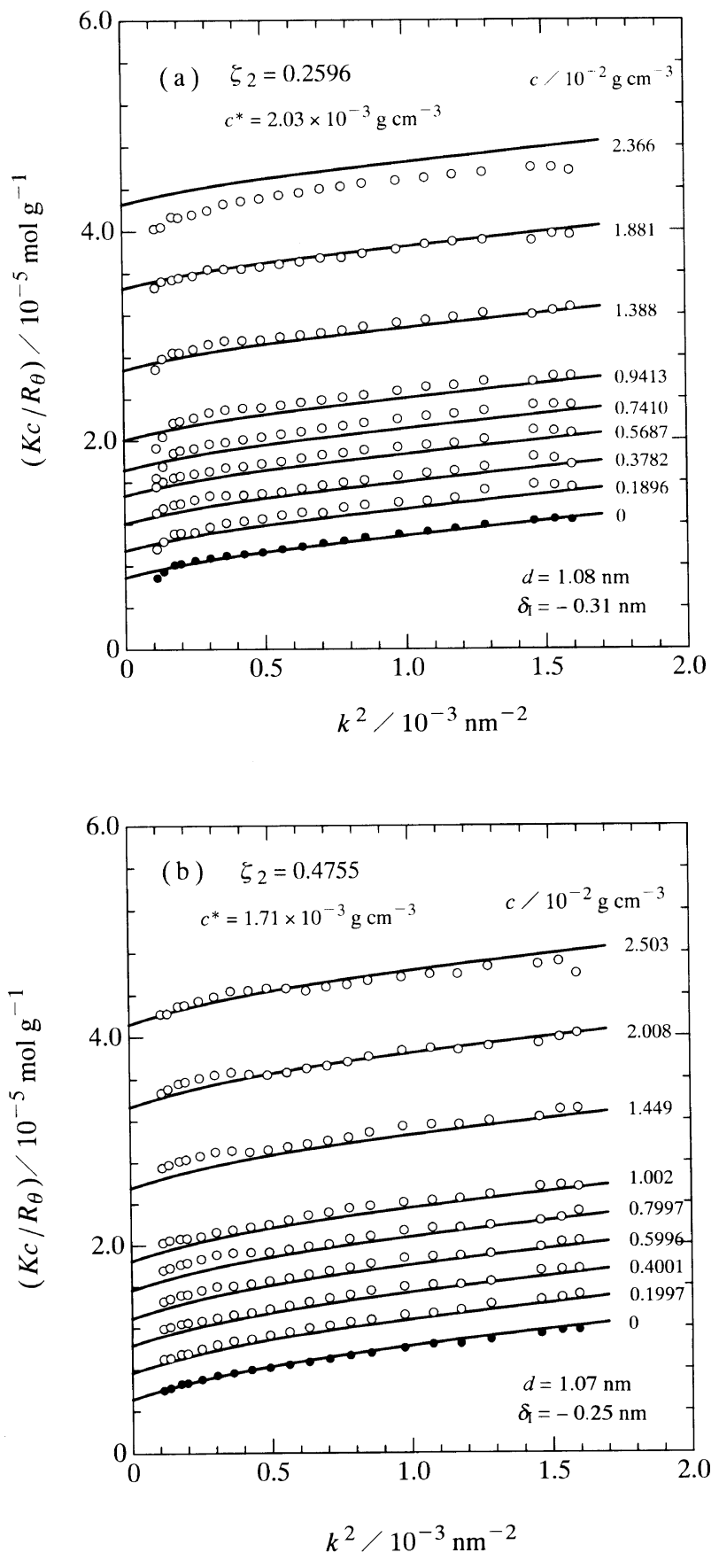

Polym. J., Vol. 31, No. 3, 1999

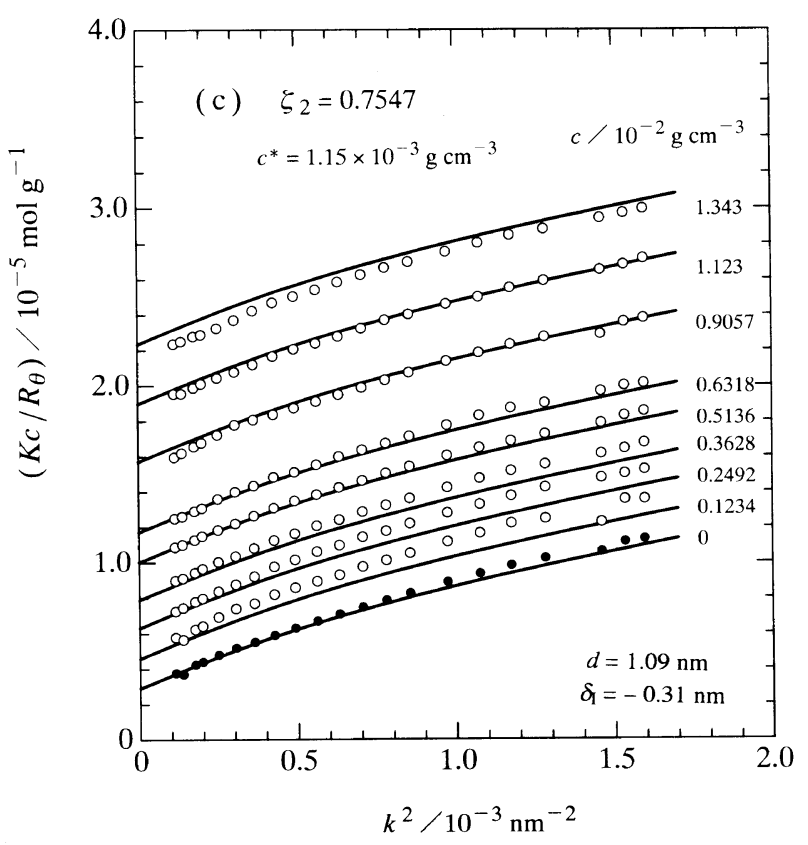

Figure 1. Angular dependence of $K c / R_{\theta}$ for dichrolomethane solutions containing two poly( $n$-hexyl isocyanate) samples of different molecular weights with three different mixing ratios; $\zeta_{2} \equiv w_{2} M_{w, 2} /$ $\left(w_{1} M_{w, 1}+w_{2} M_{w, 2}\right)$ with $M_{w, 1}=1.11 \times 10^{5}$ and $M_{w, 2}=1.06 \times 10^{6} ; c^{*}$ (the overlap concentration $)=\left(3 / 4 \pi N_{\mathrm{A}}\right) \times\left[\left(\left\langle S^{2}\right\rangle_{1}^{3 / 2} / M_{1}\right) w_{1}+\left(\left\langle S^{2}\right\rangle_{2}^{3 / 2} /\right.\right.$ $\left.\left.M_{2}\right) w_{2}\right]^{-1}$ with $\left\langle S^{2}\right\rangle_{1}^{1 / 2}=26.6 \mathrm{~nm}$ and $\left\langle S^{2}\right\rangle_{2}^{1 / 2}=97.9 \mathrm{~nm}$; solid curves, calculated from eq 22 with $M_{\mathrm{L}}=740 \mathrm{~nm}^{-1}, q=21 \mathrm{~nm}$, and values of $d$ and $\delta_{\mathrm{I}}$ given in each panel.

mass per unit contour length $M_{\mathrm{L}}$. For PHIC in DCM at $20^{\circ} \mathrm{C}, M_{\mathrm{L}}=740 \mathrm{~nm}^{-1}$ and $q=21 \mathrm{~nm}$, which were determined from the molecular weight dependence of the intrinsic viscosity and radius of gyration for PHIC in DCM. ${ }^{12,28}$ Thus we use those parameter values and search for the interaction parameters $d$ and $\delta_{1}$ leading to the best fit of the $K c / R_{\theta}$ data shown in Figure 1 to eq 22, where $P_{1}(k)$ and $P_{2}(k)$ are calculated by the theory of Yoshizaki and Yamakawa, ${ }^{18,29}$ and $\Gamma_{s t}(s, t=1$ and 2) are estimated from eq 4 along with the parameters listed in Table I. The fitting results are shown by the solid curves in Figure 1, which are obtained by selecting $d$ and $\delta_{\mathrm{I}}$ values given in each panel. The agreement between the theory and experiment is almost satisfactory. Furthermore, the values of $d$ and $\delta_{1}$ selected are close to those obtained previously from the concentration 


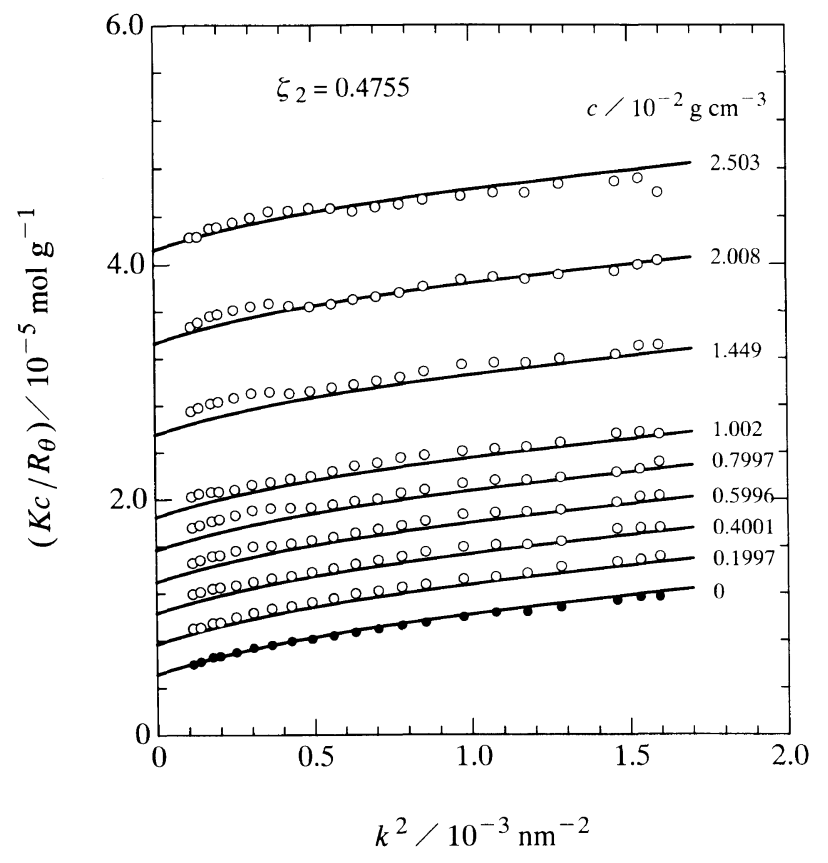

Figure 2. Comparisons of experimental results of $K c / R_{\theta}$ for the ternary solutions of PHIC $\left(\zeta_{2}=0.4755\right)$ with eq 25 and 26 , using the molecular parameters same as in Figure $1 \mathrm{~b}$.

dependence of the osmotic compressibility for binary solutions of PHIC and DCM $\left(d=1.07 \mathrm{~nm}\right.$ and $\delta_{1}=$ $-0.36 \mathrm{~nm}) .^{12}$

As mentioned in the previous section, if both $L_{1} / d$ and $L_{2} / d$ are sufficiently larger than unity, eq 22 can be approximated by a much simpler eq 25 with eq 24 . Figure 2 compares the experimental results of $K c / R_{\theta}$ for ternary PHIC solutions with $\zeta_{2}=0.4755$ with the values calculated from eq 25 and 24 with the molecular parameters same as in Figure $1 \mathrm{~b}$. The theoretical curves closely fit to the experimental data points; similar good fitting was obtained for ternary solutions with $\zeta_{2}=0.2596$ and 0.7547 . Thus we can say that the polymer-end effect on $K c / R_{\theta}$ is not important for the present ternary PHIC solutions. This effect was also unimportant on the second virial coefficient of PHIC in DCM. ${ }^{10,12}$

Figure 3 compares the same experimental data of $K c /$ $R_{\theta}$ with theoretical results calculated by eq 27 and 28 simplified using the second virial approximation. The agreement between experiment and theory is not good at high concentrations, demonstrating that the second virial approximation is not good for the present ternary PHIC solutions with $c$ much higher than the overlap concentration $c^{*}$ indicated in Figure $1 \mathrm{~b}$.

Acknowledgment. This work was supported by a Grant-in-Aid for Scientific Research (No. 06403027) from the Ministry of Education, Science, Sports and Culture of Japan and also by the Mitsubishi Chemical Corporation Fund. Y. J. gratefully acknowledges the financial support of a postdoctoral fellowship from Japan Society for Promotion of Science.

\section{APPENDIX. FORMULATION OF THE SOFT DISPERSION INTERACTION}

Let $\boldsymbol{\alpha}_{s}$ be the local excess polarizability tensor per unit

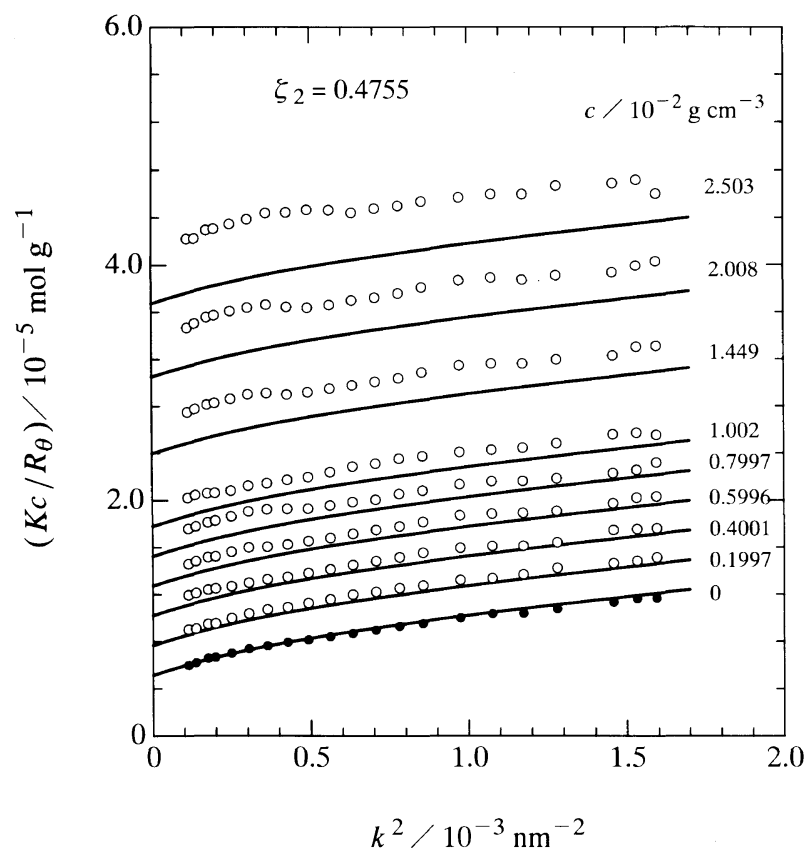

Figure 3. Comparisons of experimental results of $K c / R_{\theta}$ for the ternary solutions of PHIC $\left(\zeta_{2}=0.4755\right)$ with eq 27 and 28 , using the molecular parameters same as in Figure $1 b$.

length of a wormlike spherocylinder (WSC) of species $s$ in a solvent with the dielectric permittivity $\varepsilon$. The tensor $\alpha_{s}$ is assumed to be cylindrically symmetric; $\alpha_{s, 1}\left(=\alpha_{s, 2}\right)$ and $\alpha_{s, 3}$ denote the local excess polarizabilities parallel and perpendicular to the cylinder axis, respectively.

According to the theories of McLachlan ${ }^{30,31}$ and Imura and Okano, ${ }^{32}$ the local dispersion energy (or the van der Waals free energy) $G_{s t}\left(s_{a}, s_{b}\right)$ between two unit-length portions of WSCs $a$ and $b$ of species $s$ and $t$, respectively, on the contour points $s_{a}$ and $s_{b}$ is written as

$$
G_{s l}\left(s_{a}, s_{b}\right)=-\left(k_{\mathrm{B}} T / x^{6}\right) \sum_{k, l=1}^{3} J_{s t, k l} c_{k l}^{2}
$$

Here $x$ is the distance between the contour points $s_{a}$ and $s_{b}$, and

$$
J_{s t, k l}=\sum_{n=0}^{\prime} \alpha_{s, k}\left(i \omega_{n}\right) \alpha_{t, l}\left(i \omega_{n}\right) / \varepsilon^{2}\left(i \omega_{n}\right)
$$

where $i \omega_{n} \equiv 2 \pi i n k_{\mathrm{B}} T / \hbar$ is the imaginary frequency with the Planck constant $\hbar$, and the prime over the summation indicates that the $n=0$ term is multiplied by $1 / 2$. The coupling constant $c_{k l}$ is defined by

$$
c_{k l}=\boldsymbol{a}_{k}\left(s_{a}\right) \cdot \boldsymbol{a}_{l}\left(s_{b}\right)-3\left[\boldsymbol{a}_{k}\left(s_{a}\right) \cdot \boldsymbol{e}\right]\left[\boldsymbol{a}_{l}\left(s_{b}\right) \cdot \boldsymbol{e}\right]
$$

where $\boldsymbol{a}_{k}\left(s_{a}\right)\left(\boldsymbol{a}_{l}\left(s_{b}\right)\right)$ is a unit vector along the principal axis $k(l)$ at the contour point $s_{a}\left(s_{b}\right)$, and $e$ is a unit vector directed from the contour point $s_{a}$ to the point $s_{b}$. Assuming the pairwise additivity of the dispersion energy, we can calculate the total dispersion energy $w_{s t}$ between WSCs of species $s$ and $t$ by

$$
w_{s t}=\int_{-L_{s} / 2}^{L_{s} / 2} d s_{a} \int_{-L_{t} / 2}^{L_{t} / 2} d s_{b} G_{s t}\left(s_{a}, s_{b}\right)
$$

where $L_{s}$ and $L_{t}$ are the contour lengths of the WSC of species $s$ and $t$, respectively. Since $G_{s t}\left(s_{a}, s_{b}\right)$ is defined in solution, $w_{s t}$ should be regarded as the potential of mean 
force.

Let us first consider two long WSCs of species $s$ and $t$. We assume that the persistence lengths $q_{s}$ and $q_{t}$ of the WSCs are much longer than the interaction range of $G_{s t}\left(s_{a}, s_{b}\right)$. [It is noted that $G_{s t}\left(s_{a}, s_{b}\right)$ is a short range function of $x$ from eq A1.] In such a case, two or more pairs of contour portions on the two interacting WSCs seldom come close within the interaction range simultaneously. Therefore, it is enough to consider only the case that the two WSCs interact with each other at one pair of contour portions (the single contact approximation). As a result, $w_{\text {st }}$ calculated from eq A4 may be approximated by that for corresponding straight spherocylinders.

The binary cluster integral $\beta_{\mathrm{w}, s t}$ with respect to $w_{s t}$ for two long stiff WSCs may be written as ${ }^{12}$

$$
\beta_{\mathrm{w}, s t}=\frac{\pi}{2} L_{s} L_{t} \delta_{s t}
$$

with

$$
\delta_{s t} \equiv \frac{4}{\pi} \int_{\left(d_{s}+d_{t}\right) / 2}^{\infty}\left\langle\frac{w_{s t, \infty}}{k_{\mathrm{B}} T}|\sin \gamma|\right\rangle \mathrm{d} r
$$

where $r$ is the shortest distance between the two spherocylinders, $\gamma$ is the angle formed by the two tangent vectors of the spherocylinders at the closest contour points, $w_{s t, \infty}$ is $w_{s t}$ for infinitely long spherocylinders of species $s$ and $t$, and the angular brackets represent the orientational average of the two chains; one should not confuse $\delta_{s t}$ with the Kronecker delta. (In eq A6, we used the high-temperature approximation for the Mayer function, and also assumed $w$ to be zero at $r<d$.) We can calculate $w_{\text {st, } \infty}$ from eq A4, where $x$ and $e$ depend on $s_{a}$ and $s_{b}$, but $\boldsymbol{a}_{k}\left(s_{a}\right)$ and $\boldsymbol{a}_{l}\left(s_{b}\right)$ do not depend on $s_{a}$ and $s_{b}$. The final result is written as

$$
w_{s t, \infty}=-\frac{k_{\mathrm{B}} T}{r^{4}|\sin \gamma|}\left[J_{s t, \mathrm{I}}+J_{s t, \mathrm{~A}} P_{2}(\cos \gamma)\right]
$$

where, $P_{2}$ is the second Legendre polynomial, and

$$
\begin{aligned}
& J_{s t, \mathrm{I}} \equiv \frac{3 \pi}{32}\left(19 J_{s t, 11}+10 J_{s t, 13}+3 J_{s t, 33}\right), \\
& J_{s t, \mathrm{~A}} \equiv \frac{\pi}{8}\left(J_{s t, 11}-2 J_{s t, 13}+J_{s t, 33}\right)
\end{aligned}
$$

which depend on $\alpha$ and $\varepsilon$. Using eq A7, $\delta_{s t}$ is calculated to be

$$
\delta_{s t}=\delta_{s t, \mathrm{I}}+\delta_{s t, \mathrm{~A}} \eta_{s t}
$$

with

$$
\begin{aligned}
& \delta_{s t, \mathrm{I}} \equiv-32 J_{s t, \mathrm{I}} / 3 \pi\left(d_{s}+d_{t}\right)^{3}, \\
& \delta_{s t, \mathrm{~A}} \equiv-32 J_{s t, \mathrm{~A}} / 3 \pi\left(d_{s}+d_{t}\right)^{3}
\end{aligned}
$$

and

$$
\eta_{s t} \equiv \iint \mathrm{d} \boldsymbol{a} \mathrm{d} \boldsymbol{a}^{\prime} P_{2}\left(\boldsymbol{a} \cdot \boldsymbol{a}^{\prime}\right) \bar{f}_{s}(\boldsymbol{a}) \bar{f}_{t}\left(\boldsymbol{a}^{\prime}\right)
$$

where $\bar{f}_{s}(\boldsymbol{a})$ and $\bar{f}_{t}(\boldsymbol{a})$ are the orientational distribution functions of the unit tangent vector $\boldsymbol{a}$ to the wormlike chain contour for species $s$ and $t$, respectively.

The integral in eq $\mathrm{A} 4$ for $w_{s t}$ cannot be expressed in an analytical form when $L_{\mathrm{c}, s}$ and $L_{\mathrm{c}, t}$ are finite, and thus it is not easy to incorporate the end effect of spherocylinders on $\beta_{\mathrm{w}, s t}$. Here we treat the end effect, according to Sato and Teramoto ${ }^{33}$ who previously considered the same problem for stiff polyelectrolytes. They approximated the electrostatic interaction potential of a stiff polyelectrolyte by a square-well potential to calculate the electrostatic binary cluster integral. Similarly, we approximate $w_{s t}$ of spherocylinders with a soft dispersion potential by the following square-well potential:

$$
w_{s t}= \begin{cases}w_{s t}^{\circ}, & \left(d_{s}+d_{t}\right) / 2 \leq r \leq\left(d_{s}+d_{t}\right) / 2+\Delta_{s t} \\ 0, & \text { otherwise }\end{cases}
$$

where $w_{s t}^{\circ}$ and $\Delta_{s t}$ are two constants. When the solution is isotropic, this potential yields

$$
\beta_{\mathrm{w}, s t}=\frac{\pi}{2}\left[L_{\mathrm{c}, s} L_{\mathrm{c}, t}+2\left(L_{\mathrm{c}, s}+L_{\mathrm{c}, t}\right)\left(d_{s}+d_{t}\right)+2\left(d_{s}+d_{t}\right)^{2}\right] \delta_{\mathrm{I}, s t}
$$

where $\delta_{\mathrm{I}, s t} \equiv w_{s t}{ }^{\circ} \Delta_{s t} / k_{\mathrm{B}} T$, and terms of order of $\delta_{\mathrm{I}, s t} /$ $\left(d_{s}+d_{t}\right)$ were omitted. ${ }^{3}$ This equation reduces to eq A5 in the isotropic state, when both $L_{\mathrm{c}, s}$ and $L_{\mathrm{c}, t}$ are much larger than $d_{s}+d_{t}$. Although Sato and Teramoto calculated the electrostatic cluster integral also in an anisotropic state, we do not discuss the end effect on the anisotropic part of $\beta_{\mathrm{w} . s t}$ in this paper.

\section{REFERENCES}

1. M. B. Huglin, Ed., "Light Scattering from Polymer Solutions," Academic Press, London \& New York, 1972.

2. H. Yamakawa, "Modern Theory of Polymer Solutions," Harper \& Row, New York, N.Y., 1971.

3. J. G. Kirkwood and R. J. Goldberg, J. Chem. Phys., 18, 54 (1950).

4. W. H. Stockmayer, J. Chem. Phys., 18, 58 (1950).

5. E. F. Casassa and H. Eisenberg, J. Phys. Chem., 64, 753 (1960)

6. E. F. Casassa and H. Eisenberg, J. Phys. Chem., 65, 427 (1961).

7. H. Eisenberg, J. Chem. Phys., 36, 1837 (1962).

8. H. Yamakawa, J. Chem. Phys., 46, 973 (1967).

9. T. Sato, Y. Jinbo, and A. Teramoto, Polym. J., 27, 384 (1995).

10. T. Sato, Y. Jinbo, and A. Teramoto, Macromolecules, 30, 590 (1997).

11. M. Kurata, "Thermodynamics of Polymer Solutions," Harwood Academic Publishers, Chur, 1982.

12. Y. Jinbo, T. Sato, and A. Teramoto, Macromolecules, 27, 6080 (1994).

13. T. Sato, T. Shoda, and A. Teramoto, Macromolecules, 27, 164 (1994).

14. P. Sharp and V. A. Bloomfield, Biopolymers, 6, 1201 (1968).

15. J. des Cloizeaux, Macromolecules, 6, 403 (1973)

16. R. Koyama, J. Phys. Soc. Jpn., 34, 1029 (1973).

17. T. Norisuye, M. Motowoka, and H. Fujita, Macromolecules, 11, 966 (1978).

18. T. Yoshizaki and H. Yamakawa, Macromolecules, 13, 1518 (1980).

19. K. S. Schweizer and J. G. Curro, Phys. Rev. Lett., 58, 246 (1987).

20. J. G. Curro and K. S. Schweizer, Macromolecules, 20, 1928 (1987).

*3 Previously, we proposed a different expression of $\beta_{w}$ for polymer chains whose end portions possess different interaction properties from those of the middle portion. Equation A13 does not consider such a difference. 
21. J. G. Curro and K. S. Schweizer, J. Chem. Phys., 87, 1842 (1987).

22. K. G. Honnell, J. G. Curro, and K. S. Schweizer, Macromolecules, 23, 3496 (1990).

23. K. S. Schweizer and J. G. Curro, Phys. Rev. Lett., 60, 809 (1988).

24. K. S. Schweizer and J. G. Curro, J. Chem. Phys., 91, 5059 (1989).

25. T. Norisuye and H. Fujita, Polym. J., 14, 143 (1982).

26. C. G. Gray and K. E. Gubbins, "Theory of Molecular Fluids,"
Vol 1, Fundamentals, Clarendon Press, Oxford, 1984

27. H. Benoit and M. Benmouna, Macromolecules, 17, 535 (1984).

28. T. Itou, H. Chikiri, A. Teramoto, and S. M. Aharoni, Polym. J., 20, 143 (1988)

29. H. Yamakawa, "Helical Wormlike Chains in Polymer Solutions," Springer-Verlag, Berlin \& Heidelberg, 1997.

30. A. D. McLachlan, Proc. R. Soc. London, A271, 387 (1963).

31. A. D. McLachlan, Proc. R. Soc. London, A274, 80 (1963).

32. H. Imura and K. Okano, J. Chem. Phys., 58, 2763 (1973).

33. T. Sato and A. Teramoto, Physica, A176, 72 (1991). 